\title{
ON THE DISCRETIZATION IN TIME OF PARABOLIC STOCHASTIC PARTIAL DIFFERENTIAL EQUATIONS
}

\author{
JACQUES PRINTEMS ${ }^{1}$
}

\begin{abstract}
We first generalize, in an abstract framework, results on the order of convergence of a semi-discretization in time by an implicit Euler scheme of a stochastic parabolic equation. In this part, all the coefficients are globally Lipchitz. The case when the nonlinearity is only locally Lipchitz is then treated. For the sake of simplicity, we restrict our attention to the Burgers equation. We are not able in this case to compute a pathwise order of the approximation, we introduce the weaker notion of order in probability and generalize in that context the results of the globally Lipschitz case.
\end{abstract}

Mathematics Subject Classification. 60H15, 60F25, 60F99, 65C20, 60H35.

Received: July 24, 2000. Revised: January 8, 2001; September 17, 2001.

\section{INTRODUCTION}

Let us introduce the abstract framework of the stochastic parabolic partial differential equation which will be treated in this paper. Let $H$ be a Hilbert space, we will consider the following evolution equation written in the abstract Ito form

$$
\mathrm{d} u+(A u+f(u)) \mathrm{d} t=\sigma(u) \mathrm{d} W,
$$

with the initial condition

$$
u(0)=u_{0} \in H,
$$

where $u$ is a $H$-valued random process, $A: D(A) \subset H \rightarrow H$ denotes an unbounded, non-negative self-adjoint operator, such that $D(A)$ is compactly embedded into $H, f$ is a non-linear mapping from $H$ into $D\left(A^{-s}\right)$ for some $s \in\left[0,1\left[\right.\right.$ and $\sigma$ is a mapping from $H$ into $\mathcal{L}\left(H, D\left(A^{-\beta}\right)\right)$, the space of linear bounded operator from $H$ into $D\left(A^{-\beta}\right)$ for some $\beta$. The interplay between $A$ and $\beta$ will be specified below. Here, $\{W(t)\}_{t \geq 0}$ denotes a cylindrical Wiener process on $H$ (see [8]) defined on a given stochastic basis $\left(\Omega, \mathcal{F}, \mathbb{P},\left\{\mathcal{F}_{t}\right\}_{t \geq 0}\right)$.

Such a framework is very general. It includes the stochastic versions of the Burgers equation, the NavierStokes equation, the Cahn-Hilliard equation, the Ginzburg-Landau equation, the Kuramoto-Sivashinsky equation or more generally reaction-diffusion equations. If theses equations are considered on a bounded domain then they fit the above framework.

Keywords and phrases. Stochastic partial differential equations, semi-discretized scheme for stochastic partial differential equations, Euler scheme.

1 Centre de Mathématiques de l'Université de Paris 12, EA 2343, Université de Paris 12, 61 avenue du Général de Gaulle, 94010 Créteil Cedex, France. e-mail: printems@univ-paris12.fr 
For example, in space dimension one, let us consider the following stochastic partial differential equations

$$
\frac{\partial u(x, t)}{\partial t}-\frac{\partial^{2} u(x, t)}{\partial x^{2}}+F(u(x, t))=G(u(x, t)) \frac{\partial^{2} \widetilde{W}(x, t)}{\partial x \partial t},
$$

or

$$
\frac{\partial u(x, t)}{\partial t}-\frac{\partial^{2} u(x, t)}{\partial x^{2}}+\frac{\partial F(u(x, t))}{\partial x}=G(u(x, t)) \frac{\partial^{2} \widetilde{W}(x, t)}{\partial x \partial t},
$$

where $x \in(0,1)$ and $t>0$, with Dirichlet boundary conditions and an initial condition.

Where $u=u(x, t)$ is a $\mathbb{R}$-valued random process and $\{\widetilde{W}(x, t)\}$ a brownian sheet on $] 0,1\left[\times \mathbb{R}_{+}\right.$. We recall that $\{\widetilde{W}(x, t)\}$ is a zero mean gaussian process such that

$$
\mathbb{E} \widetilde{W}(x, t) \widetilde{W}(y, s)=(x \wedge y)(t \wedge s),
$$

for $t, s$ and $x, y$. We recognize for example in equation (1.4), for $F(y)=y^{2} / 2$, the Burgers equation. Other boundary conditions could be considered.

In both cases, $H=L^{2}(0,1), A$ denotes the operator $-\frac{\partial^{2}}{\partial x^{2}}$ defined on $D(A)=H^{2}(0,1) \cap H_{0}^{1}(0,1)$. For equation (1.4), we set $f(u)(x)=\frac{\partial(F(u(x)))}{\partial x}$ and if for instance $F: \mathbb{R} \rightarrow \mathbb{R}$ is a Lipschitz function it is easy to check that $f$ is a Lipschitz function from $H=L^{2}(0,1)$ to $H^{-1}(0,1)=D\left(A^{-1 / 2}\right)$ so that we take $s=1 / 2$. We will see in Section 4 that for the Burgers case, $f(u)(x)=\frac{1}{2} \frac{\partial\left(u(x)^{2}\right)}{\partial x}$, we can take any $s>3 / 4$.

Also, $W(t)$ can formally be written as the space derivative of $\widetilde{W}(t, x)$. More precisely,

$$
W(t)=\frac{\partial \widetilde{W}}{\partial x}=\sum_{i \geq 0} \beta_{i}(t) e_{i},
$$

where $\left\{e_{i}\right\}_{i \geq 0}$ are the eigenfunctions of $A$ and form a Hilbertian basis of $L^{2}(0,1) ;\left\{\beta_{i}\right\}_{i \geq 1}$ is a family of real Brownian motions mutually independent in a fixed probability space. In the case of equations (1.3)-(1.4),

$$
e_{i}(x)=\sqrt{\frac{2}{\pi}} \sin (i \pi x), \quad x \in(0,1) .
$$

Also, in (1.3)-(1.4), we can consider $\sigma(u)$ to be the operator given by

$$
\begin{aligned}
\sigma(u) \quad: \quad & H \longrightarrow H \\
& v \mapsto G(u) v
\end{aligned}
$$

where $G: \mathbb{R} \rightarrow \mathbb{R}$ is a bounded function. In this case $\beta=0$. Another example is provided by the stochastic Cahn-Hilliard equation which in Ito form reads

$$
\mathrm{d} u+\left(\Delta^{2} u+\Delta f(u)\right) \mathrm{d} t=\sigma(u) \mathrm{d} W,
$$

with Neumann boundary conditions for $(x, t) \in D \times \mathbb{R}^{+}, D$ an open bounded set on $\mathbb{R}^{n}, n=1,2,3$. Here $H=L^{2}(0,1)$ and $A=\Delta^{2}$ on the domain $H^{4}(D)$. Again, if $f$ is Lipschitz we can take $s=1 / 2$. The noise term may be of the same form as described in (1.5) and then $\beta=0$. However, it is also physically relevant to consider a noise of the form $\nabla(G(u) \mathrm{d} W)$ in which case $\beta=1 / 4$. Nevertheless, some restrictions described below do not allow to treat this latter noise unless $n=1$. 
Several authors have already considered Cauchy Problems associated to (1.1)-(1.2) or (1.3)-(1.5) (see [1,8, $16,19,20,24,25]$ and references therein). Results of existence and uniqueness of global strong solutions $u$ are classical if $f$ and $\sigma$ are globally Lipschitz. In many cases where $f$ is only locally Lipschitz (stochastic Burgers equation, stochastic Cahn-Hilliard or stochastic Navier-Stokes), and $\sigma$ is bounded, existence of global strong solutions can also be shown (see $[3,5-7,9,13])$.

In this paper, we are interested in the approximation of (1.1)-(1.2) by a semi-implicit scheme. Let $T>0$ and $N$ an integer. We set $\tau=T / N$ and construct a sequence of approximations $\left\{u^{n}\right\}_{0 \leq n \leq N}$ of the solution $u$ by the following scheme:

$$
\left\{\begin{array}{l}
u^{n+1}-u^{n}+\tau\left(A u^{n+\theta}+f\left(u^{n}\right)\right)=\sqrt{\tau} \sigma\left(u^{n}\right) \chi^{n} \\
u^{0}=u_{0} \in H
\end{array}\right.
$$

where we have set $u^{n+\theta}=\theta u^{n+1}+(1-\theta) u^{n}$ for some $\theta \in[0,1]$ and $\left\{\chi^{n}\right\}_{n \geq 0}$ is a sequence of i.i.d. $H$-valued normal random variables.

Of course, (1.7) is formal and has to be understood in the following sense

$$
u^{n+1}=S_{\tau} u^{n}-(I+\tau \theta A)^{-1} f\left(u^{n}\right)+(I+\tau \theta A)^{-1} \sqrt{\tau} \sigma\left(u^{n}\right) \chi^{n},
$$

with $S_{\tau}=(I+\tau \theta A)^{-1}(I-\tau(1-\theta) A)$. Note that under our assumptions $S_{\tau}$ can be extended to an operator bounded from $H$ to $H$ and since $s<1,(I+\tau \theta A)^{-1} f\left(u^{n}\right) \in D\left(A^{1-s}\right) \subset H$ for $u^{n} \in H$.

Concerning the scheme (1.7), let us note that results on the order of convergence in the deterministic case $(\sigma=0)$ are classical. For instance, in $[4,17]$, the authors have shown that the infinite dimensional setting implies the restriction

$$
\theta>1 / 2
$$

for the convergence. In this case, they were able to show that this scheme is of order 1 in the absence of noise.

Typically, since here $u^{n}, u$ are $H$-valued random variables, various type of convergence and order can be considered, either we consider pathwise approximation (strong order) or approximation of the law (weak order). In the finite dimensional case, discretization in time of stochastic differential equations have already been discussed by several authors $[15,18,22,23]$. Typically, the lack of regularity of the noise implies that the explicit Euler scheme applied to such equations is of strong order $1 / 2$. Nevertheless, in [18], the author noted that, provided suitable assumptions on the coefficients hold and when some correction terms are introduced (which vanish for additive noise), the explicit Euler scheme is of strong order 1 and of weak order 2 (see also [22, 23]).

Available results are much weaker in the infinite dimensional case. Recently, in the case where $A$ is the Laplacian operator on the interval $(0,1)$ with Dirichlet boundary conditions, Gyöngy and Nualart [12] have been able to prove the $L^{p}(\Omega)$ convergence for any $p$ of the approximations $u^{n}$ given by (1.7) to the exact solution $u$ in the case of equation (1.3). Moreover, by the mean of the Green function, they found a rate of the convergence in $\tau^{\gamma}$ with $\gamma<1 / 4$ when $f$ and $\sigma$ are globally Lipschitz. In the case where $f$ and $\sigma$ are only locally bounded, continuous functions, they proved a convergence in probability of the approximation to the exact solution.

It seems difficult to use similar ideas as in $[18,22,23]$ and obtain better order of convergence, this would require strong assumptions on the spatial regularity of the noise.

Our first goal in this paper is to generalize the first part of [12] concerning globally Lipschitz nonlinearity. There, the authors use extensively the explicit form of the Green function of the continuous problem. Here, we propose to use spectral properties of the linear operator $A$. Thus, we can use powerful tools already developped in the deterministic case. Also, the Green function is not always available so that we think that our method applies to more situations.

The main difficulty in studying numerical schemes for stochastic partial differential equations is in the treatment of the time discretization. For the sake of simplicity, we have prefered to describe first this part. However 
as will be exposed in a forthcoming work, our arguments extend to the analysis of a fully discretized scheme when combined with the arguments used in the deterministic theory. For instance the tools introduced in [2,14] allow to study a discretization with finite elements on a non uniform grid. Note that in [12] a full discretization with finite difference is considered; there a discrete Green function is used. However, it seems that this approach is restricted to uniform grids.

In this first part, we will be able to prove the $L^{p}$-convergence of $u^{n}$ to $u$ with the rate $\widetilde{\gamma}<\min (1-s,(1-$ $\alpha) / 2-\beta)$. Our main assumptions are in this case

$$
\operatorname{Tr}\left(A^{-\alpha}\right)<+\infty
$$

for some $\alpha>0$.

This trace assumption is very natural when stochastic partial differential equations of parabolic type are concerned. It consists in assuming that the linear operator has discrete spectral values and that the $p$-th power of their inverses defines a convergent series for some $p$. This is not very restrictive when dealing with elliptic operator on a bounded domain.

The following assumptions are

$$
|f(u)-f(v)|_{D\left(A^{-s}\right)} \leq L_{f}|u-v|_{H}
$$

and

$$
|\sigma(u)-\sigma(v)|_{\mathcal{L}\left(H, D\left(A^{-\beta}\right)\right)} \leq L_{\sigma}|u-v|_{H},
$$

for any $(u, v)$ in $H$ and for some constants $L_{f}, L_{\sigma}>0$. The coefficients $\alpha$, defined by the trace assumption (1.9), and $\beta$, the space regularity of the noise, are linked by the inequality

$$
1-\alpha-2 \beta>0
$$

which is essential when proving the Hilbert-Schmidt property of the semi-group $\left\{e^{-t A}\right\}_{t \geq 0}(c f$. Lem. 2.2) in the space $\mathcal{L}\left(H, D\left(A^{-\beta}\right)\right)$. Concerning the nonlinearity, note that $s \geq 0$ allows $f$ to depend on some spatial derivatives of the solution. Moreover, we assume that $s<1$ in order that, as it is classical in the deterministic case, the following expression (and its discrete version) make sense

$$
\int_{0}^{t} e^{-(t-\tau) A} f(u(\tau)) \mathrm{d} \tau .
$$

Indeed, we can note that the roles played by the nonlinearity and the noise are independant both in the assumptions and in the expression of the rate of convergence.

In this setting, the result of this section is general and can be applied to all parabolic stochastic partial differential equations with a Lipschitz non linearity provided (1.12) holds. For example, in dimension $d$ with a white noise $(\beta=0)$, let $A=(-\Delta)^{r / 2}$ for some positive real $r>d$ on some bounded domain of $\mathbb{R}^{d}$ with Dirichlet or Neumann boundary conditions. If $\alpha$ is any number greater than $d / r$ then condition (1.9) holds. If $s=0$, we find that the order of convergence of $u^{n}$ is $\frac{1}{2}\left(1-\frac{d}{r}\right)$. For the Cahn-Hilliard equation (1.6) with a Lipschitz $f$, if $\beta=0$ we obtain an order $3 / 8$ in space dimension $1,1 / 4$ in space dimension 2 and $1 / 8$ in space dimension 3 . If $\beta=1 / 4$, condition (1.12) cannot hold if $n=2$ or 3 . And for $n=1$, we obtain an order $1 / 8$.

We then treat the case where $\sigma$ is still globally Lipschitz but the nonlinearity $f$ is only locally Lipschitz. We will restrict ourselves to the one-dimensionnal case of the stochastic Burgers equation (Eq. (1.4) with $\left.f(y)=y^{2}\right)$. In this case $H=L^{2}(0,1), s>3 / 4, \beta=0$ and $\alpha>1 / 2$. However our approach is general and, using the setting of the preceeding section, can be applied in many situations. The idea is to mimic the proof of existence and uniqueness for the continuous problem (see [7]). An extra assumption for $\sigma$ is required

$$
\forall u \in L^{2}(0,1), \quad|\sigma(u)|_{\mathcal{L}\left(L^{2}(0,1)\right)} \leq C_{\sigma},
$$


for some $C_{\sigma}>0$. We consider truncated versions of (1.1) and (1.7) for which the results of the preceeding section hold. Then, we prove convergence in probability of the scheme which implies boundedness in probability. These two facts are used together to obtain that

$$
\lim _{C \rightarrow+\infty} \mathbb{P}\left\{\tau^{-\tilde{\gamma}} \max _{0 \leq n \leq N}\left|u^{n}-u(n \tau)\right|_{H} \geq C\right\}=0,
$$

for $\widetilde{\gamma}<1 / 4$, uniformly with respect to $N$. We say that the scheme is of order in probability $\widetilde{\gamma}<1 / 4$.

This definition is quite natural. Indeed, a deterministic scheme is of order $\widetilde{\gamma}$ if

$$
\tau^{-\tilde{\gamma}} \max _{0 \leq n \leq N}\left|u^{n}-u(n \tau)\right|_{H}
$$

is bounded. Since different notions of boundedness (almost surely, in $L^{p}(\Omega, H)$, in probability) can be considered for random variables, different notions of order of convergence naturally arise.

The paper is organized as follows. In Section 2, after some notations and preliminairies, we will present the different notions of order that we will need afterwards. In Section 3, we give in Theorem 3.2 the general results in the case of a globally Lipschitz non linearity and obtain a $L^{p}$-order of convergence $\widetilde{\gamma}<\min (1-s,(1-\alpha) / 2-\beta)$. In Section 4, we will first recall existence and uniqueness of stochastic process solution of the Burgers equation (cf. [7]). We then give in Theorem 4.3 the result of order in probability of the scheme (1.7) in the case of the Burgers equation with the extra assumption (1.13).

These ideas are general and although we only consider the Burgers equation, our approach can be extended to others equations: stochastic reaction diffusion equations, stochastic Navier-Stokes equation or stochastic Cahn-Hilliard equation with a polynomial nonlinearity.

\section{Preliminaries}

\subsection{Some notations}

Let $X$ be a Banach space. We will denote by $|\cdot|_{X}$ the norm on the Banach space $X$. In the case when $X=H$ is a Hilbert space, we will simply use $|\cdot|$. If $(\Omega, \mathcal{F}, \mathbb{P})$ denotes a probability space, we will denote by $L^{p}(\Omega, X), 1 \leq p<+\infty$, the space of $X$-valued random variables $u$ with Böchner integrable $p$-th power on $\Omega$. We set

$$
\mathbb{E}|u|_{X}^{p}=\int_{\Omega}|u(\omega)|_{X}^{p} \mathbb{P}(\mathrm{d} \omega)
$$

Now let $\sigma$ be a linear operator from $F$ into $G$, two Hilbert spaces, $\sigma$ is said to be Hilbert-Schmidt if the following sum is finite:

$$
|\sigma|_{L_{2}^{0}(F, G)}^{2} \stackrel{\text { def }}{=} \sum_{i \geq 1}\left|\sigma e_{i}\right|_{G}^{2}<+\infty
$$

where $\left\{e_{i}\right\}_{i>1}$ is a Hilbertian basis of $F$ and where the set of all Hilbert-Schmidt operators is denoted by $L_{2}^{0}(F, G)$. When $F=G$, we will use the notation $L_{2}^{0}(F)$ and when $F=G=L^{2}(0,1)$, the shorter notation $L_{2}^{0}$ will be used.

Let $A$ be a positive, self-adjoint operator, unbounded on $H$, a Hilbert space. We will denote by $D(A)$, its domain. We assume furthermore that $D(A)$ is compactly embedded in $H$. It follows that there exists a series of increasing real numbers $\left\{\lambda_{i}\right\}_{i \geq 1}$ such that

$$
0 \leq \lambda_{1} \leq \lambda_{2} \leq \cdots \leq \lambda_{i} \longrightarrow+\infty,
$$

which represents the eigenvalues of $A$. Moreover, we suppose that there exist a real positive $\alpha$ such that

$$
\sum_{i \geq 1} \lambda_{i}^{-\alpha}<+\infty
$$


Equation (2.1) is the translation of the trace assumption (1.9). Let us recall that, as written above in the introduction after (1.9), such assumptions are very general when bounded domains are concerned in the parabolic context. For example, (2.1) holds when the linear operator is the realization of the Laplacian on a bounded domain with Dirichlet or Neuman boundary conditions. More generally, other elliptic operators can be considered.

A family of eigenvectors $\left\{e_{i}\right\}_{i \geq 1}, e_{i} \in H$ is associated to these eigenvalues. It constitutes a Hilbertian basis of $H$. For any $u=\sum_{i \geq 1} u_{i} e_{i}$ in $D(A)$, we get

$$
A u=\sum_{i \geq 1} \lambda_{i} u_{i} e_{i}
$$

We can define for any $s \in \mathbb{R}$, the operator $A^{s}$ and its domain $D\left(A^{s}\right)$. We have for any $u \in D\left(A^{s}\right)$

$$
A^{s} u=\sum_{i \geq 1} \lambda_{i}^{s} u_{i} e_{i}
$$

and

$$
D\left(A^{s}\right)=\left\{u=\sum_{i \geq 1} u_{i} e_{i} \quad \text { such that }|u|_{D\left(A^{s}\right)}^{2} \stackrel{\text { def }}{=}\left|A^{s} u\right|_{H}^{2}=\sum_{i \geq 1} \lambda_{i}^{2 s} u_{i}^{2}<+\infty\right\} .
$$

It is well known that $A$ generates a contraction semi-group on $H$ which will be denoted by $\left\{e^{-t A}\right\}_{t \geq 0}$. The main properties of such a semi-group which will be used throughout this paper are summarized in the following lemmas.

Lemma 2.1. Let $a \geq 0$, then

$$
\begin{gathered}
\forall t \geq 0, \quad\left|e^{-t A}\right|_{\mathcal{L}(H)} \leq 1, \\
\forall t>0, \quad\left|e^{-t A}\right|_{\mathcal{L}\left(H, D\left(A^{a}\right)\right)} \leq C_{a} t^{-a} .
\end{gathered}
$$

Moreover, for $b \in[0,1]$,

$$
\left|A^{-b}\left(I d_{H}-e^{-t A}\right)\right|_{\mathcal{L}(H)} \leq C_{b} t^{b}
$$

Eventually, (2.1) will strengthen the property (2.5) as shown in the following result whose elementary proof is left to the reader.

Lemma 2.2. For any $t>0$ and for any $\beta<(1-\alpha) / 2, e^{-t A}$ is Hilbert-Schmidt from $H$ into $D\left(A^{\beta}\right)$. More precisely, for any $\widetilde{\gamma}<\gamma$, there exists $C_{\gamma}>0$ such that

$$
\forall t>0, \quad\left|e^{-t A}\right|_{L_{2}^{0}\left(H, D\left(A^{\beta}\right)\right)} \leq C_{\bar{\gamma}}\left(\sum_{i \geq 1} \lambda_{i}^{-\alpha-(\gamma-\bar{\gamma})}\right)^{1 / 2} t^{(\bar{\gamma}-1) / 2}
$$

where $\gamma=1-\alpha-2 \beta>0$.

We will need below the following discrete version of the well-known Gronwall Lemma: 
Lemma 2.3. Let $\tau$ be a positive number and $\left\{\xi_{n}\right\}_{n \geq 0},\left\{\eta_{n}\right\}_{n \geq 0}$ be two sequences of nonnegative real numbers such that $\xi_{0}=\eta_{0}=0$ and such that there exists a positive constant $L$ such that

$$
\forall n \geq 1, \quad \xi_{n} \leq L \sum_{k=0}^{n-1} \xi_{k}+\eta_{n} .
$$

Then, we have

$$
\forall n \geq 1, \quad \xi_{n} \leq \sum_{k=0}^{n-1} e^{(n-k-1) L}\left(\eta_{k+1}-\eta_{k}\right) .
$$

Proof. By induction with respect to $n$ using $L \leq e^{L}-1$.

Remark 2.4. From the Lemma 2.3, we can deduce that

$$
\forall n \geq 1, \quad \xi_{n} \leq e^{(n-1) L} \eta_{n} .
$$

\subsection{Definitions of different notions of order}

We now give the definitions of orders of convergence for a numerical scheme which will be used in Section 3 and 4 . We first note that since we consider random variables, different notions of convergence can be taken into account. Let $u^{n}$ be a sequence of $H$ valued random variable defined by some numerical scheme $\Sigma$ and $u$ be a $H$-valued stochastic process on the time interval $[0, T]$. Let $\tau=T / N$ where $N$ is an integer. We say that $u^{n}$ converge almost surely to $u$ if

$$
\lim _{N \rightarrow+\infty} \max _{0 \leq n \leq N}\left|\left(u^{n}-u(n \tau)\right)(\omega)\right|_{H}=0, \quad \text { a.s. } \omega .
$$

Also we will say that the scheme $\Sigma$ is convergent in $L^{p}(\Omega, H)$ for some $p \geq 1$ if

$$
\lim _{N \rightarrow+\infty} \mathbb{E} \max _{0 \leq n \leq N}\left|\left(u^{n}-u(n \tau)\right)\right|_{H}^{p}=0 .
$$

Finally, we define convergence in probability of $\Sigma$ when

$$
\forall \varepsilon>0, \lim _{N \rightarrow+\infty} \mathbb{P}\left\{\max _{0 \leq n \leq N}\left|\left(u^{n}-u(n \tau)\right)\right|_{H} \geq \varepsilon\right\}=0 .
$$

These three kinds of convergence are somewhat pathwise notions. At the opposite, in the case of convergence in law of the sequence $u^{n}$, we say that there is weak convergence:

$$
\lim _{N \rightarrow+\infty} \max _{0 \leq n \leq N} \mathbb{E}\left(\varphi\left(u^{n}\right)-\varphi(u(n \tau))\right)=0,
$$

for any real valued measurable bounded function $\varphi$ defined on $H$.

These notions of convergence are connected with corresponding notions of order.

Definition 2.5 (a.s. order). Let $X$ be a Banach space. Let $\left\{u^{n}\right\}_{0 \leq n \leq N}$ a sequence of $X$-valued random variables defined by a numerical scheme $\Sigma$ and $u$ a $X$-valued stochastic process on the time interval $[0, T]$. We say that the scheme $\Sigma$ is of a.s. order $\widetilde{\gamma}>0$ in $X$ if for a.s. $\omega$, there exists a constant $C(\omega)>0$ such that

$$
\max _{0 \leq n \leq N}\left|u^{n}-u(n \tau)\right|_{X} \leq C(\omega) \tau^{\bar{\gamma}}
$$

for any $N \geq 1$. 
Definition 2.6 ( $L^{p}$ order). Let $X$ be a Banach space and $\left\{u^{n}\right\}_{0 \leq n \leq N}$ a sequence of $X$-valued random variables defined by a scheme $\Sigma$ and $u$ a $X$-valued stochastic process on the time interval $[0, T]$. Let $p \geq 1$ be an integer. We say that the scheme $\Sigma$ is of $L^{p}$ order $\widetilde{\gamma}>0$ in $X$ if there exists a constant $C(p)>0$ such that

$$
\left(\mathbb{E} \max _{0 \leq n \leq N}\left|u^{n}-u(n \tau)\right|_{X}^{p}\right)^{1 / p} \leq C(p) \tau^{\bar{\gamma}}
$$

for any $N \geq 1$.

The following notion is weaker than the previous two but is still connected with pathwise approximation and corresponds to the convergence in probability (2.10):

Definition 2.7 (Order in probability). Let $X$ be a Banach space. Let $\left\{u^{n}\right\}_{0 \leq n \leq N}$ a sequence of $X$-valued random variables defined by a numerical scheme $\Sigma$ and $u$ a $X$-valued stochastic process on the time interval $[0, T]$. We will say that the scheme $\Sigma$ is of order in probability $\widetilde{\gamma}>0$ in $X$, if

$$
\lim _{C \rightarrow+\infty} \mathbb{P}\left\{\max _{0 \leq n \leq N}\left|u^{n}-u(n \tau)\right|_{X} \geq C \tau^{\tilde{\gamma}}\right\}=0,
$$

uniformly with respect to $\tau$.

The connections between these notions of order can be summarized as follows

Lemma 2.8. Let $u^{n}$ a sequence of $H$-valued random variables defined by some scheme $\Sigma$. Let $u$ be a $H$-valued stochastic process on the interval $[0, T]$. Then, the following assertions hold

1. If $\Sigma$ is of $L^{p}$-order $\widetilde{\gamma}$ with $p>1 / \widetilde{\gamma}$, then $\Sigma$ is of order in probability $\widetilde{\gamma}$;

2. If $\Sigma$ is of $L^{p}$-order $\widetilde{\gamma}$ in $H$ with $p>1 / \widetilde{\gamma}$, then $\Sigma$ is of a.s. order $\widetilde{\gamma}^{\prime}<\widetilde{\gamma}-\frac{1}{p}$;

3. If $\Sigma$ is of a.s. order $\widetilde{\gamma}$ then $\Sigma$ is of order in probability $\widetilde{\gamma}$;

4. If $\Sigma$ is of $L^{p}$-order $\widetilde{\gamma}>0$ for any $p \geq 2$, then $\Sigma$ is of a.s. order $\widetilde{\gamma}^{\prime}<\widetilde{\gamma}$.

Proof.

1. It is a consequence of the Bienaymé-Tchebyschev inequality.

2. It can be proved using the Borel-Cantelli lemma.

3. It is the Egorov lemma.

4. This assertion comes from the second one.

\section{The globally Lipschitz CASE}

\subsection{Setting of the problem and main result}

We recall here some results on the continuous Cauchy problem which is written in the Ito form

$$
\mathrm{d} u+(A u+f(u)) \mathrm{d} t=\sigma(u) \mathrm{d} W,
$$

supplemented with the initial condition

$$
u(0)=u_{0} \in H .
$$

Here, $\{W(t)\}_{t>0}$ will denote a cylindrical Wiener process defined on the Hilbert space $H$ and $A$ is the operator introduced in Section 2. 
The nonlinearity is denoted by $f$, a mapping from $H$ into $D\left(A^{-s}\right)$, for some $\left.s \in\right] 0,1[$, and $\sigma$ is considered as an application from $H$ into $\mathcal{L}\left(H, D\left(A^{-\beta}\right)\right)$ where $\beta$ is such that

$$
\exists \gamma>0, \quad \sum_{i \geq 1} \lambda_{i}^{\gamma+2 \beta-1}<+\infty .
$$

We note that thanks to (2.1), (3.3) holds as soon as we have

$$
\beta<\frac{1-\alpha}{2} \text {. }
$$

We assume furthermore that there exist real numbers $L_{f}, L_{\sigma}>0$ such that

$$
\begin{gathered}
\left|A^{-s}(f(u)-f(v))\right| \leq L_{f}|u-v|, \\
\left|A^{-s} f(u)\right| \leq L_{f}(1+|u|) \\
\left|A^{-\beta}(\sigma(u)-\sigma(v))\right|_{\mathcal{L}(H)} \leq L_{\sigma}|u-v|, \\
\left|A^{-\beta} \sigma(u)\right| \leq L_{\sigma}(1+|u|) .
\end{gathered}
$$

for any $u, v$ in $H$.

Then we have the following result of existence whose proof, which consists in a contraction argument in $L^{\infty}\left([0, T], L^{p}(\Omega, H)\right)$, can be found in Theorem 7.4, p. 186 in [8].

Proposition 3.1. Let $T>0$ and $p$ an integer such that $p \geq 2$. Then, under the assumptions (3.3)-(3.7), for any $u_{0} \in L^{p}(\Omega, H)$, there exists a unique stochastic process $u$ solution of the Cauchy problem (3.1)-(3.2).

Moreover, there exists some constants $C(T)>0$ such that

$$
\sup _{t \in[0, T]}\left(\mathbb{E}|u(t)|^{p}\right)^{1 / p} \leq C(T)\left(1+\left(\mathbb{E}\left|u_{0}\right|^{p}\right)^{1 / p}\right) .
$$

Now, assume that we are given a subdivision of the interval $[0, T]$ with uniform time step $\tau$ where $\tau=T / N$ for an integer $N$. We seek an approximation of the values $\{u(n \tau)\}_{0 \leq n \leq N}$ where $u$ is the solution of (3.1)-(3.2) on the interval $[0, T]$. The following scheme is choosen

$$
u^{n+1}=u^{n}-\tau\left(A u^{n+\theta}+f\left(u^{n}\right)\right)+\sqrt{\tau} \sigma\left(u^{n}\right) \chi^{n}
$$

where $u^{n+\theta}=\theta u^{n+1}+(1-\theta) u^{n}$ for $\theta$ which will be precised below and where $\left\{\chi^{n}\right\}_{0 \leq n \leq N}$ denotes a sequence of independent normal random variables $\left(\chi^{n}=\frac{1}{\sqrt{\tau}}(W((n+1) \tau)-W(n \tau))\right)$. Scheme (3.9) is formal and has to be understood in the following sense

$$
u^{n+1}=S_{\tau} u^{n}-(I+\tau \theta A)^{-1} f\left(u^{n}\right)+(I+\tau \theta A)^{-1} \sqrt{\tau} \sigma\left(u^{n}\right) \chi^{n},
$$

with $S_{\tau}=(I+\tau \theta A)^{-1}(I-\tau(1-\theta) A)$ bounded from $H$ to $H$.

We assume furthermore that

$$
\theta>\frac{1}{2}
$$


We wish to understand how $u^{n}$ approximates $u(n \tau)$. More precisely, we set for any $n \leq N$

$$
e^{n}=u^{n}-u(n \tau) \text {. }
$$

The main result of this section is stated below.

Theorem 3.2. Let $T>0$ and $u_{0} \in L^{p}(\Omega, H)$ for some integer $p \geq 2$. Let $u$ be the solution of (3.1)-(3.2) on [0,T] given by Proposition 3.1.

Then under assumptions (3.3)-(3.7) and (3.10), for any $\widetilde{\gamma}<\min \{1-s,(1-\alpha) / 2-\beta\}$, the scheme (3.9) is of $L^{p}$-order $\widetilde{\gamma}$ in $H$. More precisely, there exists a constant $C(p)>0$ such that

$$
\left(\mathbb{E}\left|e^{n}\right|^{p}\right)^{1 / p} \leq C(p)\left(\tau^{\bar{\gamma}}+\frac{1}{n}\left(\mathbb{E}\left|u_{0}\right|^{p}\right)^{1 / p}\right),
$$

for any $n \in\{1, \ldots, N\}$.

Remark 3.3. The term $\frac{1}{n}\left(\mathbb{E}\left|u_{0}\right|^{p}\right)^{1 / p}$ involves a term of order 1 in $\tau$ except for small times. Indeed, we have

$$
\frac{1}{n}\left(\mathbb{E}\left|u_{0}\right|^{p}\right)^{1 / p}=\frac{\tau}{n \tau}\left(\mathbb{E}\left|u_{0}\right|^{p}\right)^{1 / p} \leq \tau\left(\mathbb{E}\left|u_{0}\right|^{p}\right)^{1 / p},
$$

for $n \tau \geq 1$ for example. Hence, since $\widetilde{\gamma}<1$, the estimate of Theorem 3.2 becomes

$$
\left(\mathbb{E} \max _{n^{\star} \leq n \leq N}\left|e^{n}\right|^{p}\right)^{1 / p} \leq C \tau^{\bar{\gamma}}
$$

where $n^{\star}=E(1 / \tau)+1$. This component of the error is purely deterministic.

\subsection{Regularity in time of the solution of (3.1)-(3.2) with values in $H$}

As in the deterministic theory, in order to prove Theorem 3.2, we need regularity in time of the continuous solution. It is the purpose of this subsection. The proof of Theorem 3.2 is postponed to Section 3.3 while the proof of the following Proposition can be found in appendix.

Proposition 3.4. Let $u$ be a solution of (3.1)-(3.2). Then according to (3.3)-(3.7), for any $\left(t_{1}, t_{2}\right) \in[0, T]^{2}$, for any $p \geq 2$ and $\widetilde{\gamma}<\min \{(1-\alpha) / 2-\beta, 1-s\}$, we have

$$
\mathbb{E}\left|u\left(t_{1}\right)-u\left(t_{2}\right)\right|^{p} \leq C(p, T, f, \sigma)\left(1+\sup _{t \in[0, T]} \mathbb{E}|u(t)|^{p}\right)\left|t_{1}-t_{2}\right|^{\mid \gamma^{p}} .
$$

Corollary 3.5. Let $u$ be the solution of (3.1)-(3.2) given by Proposition 3.1, then there exists a version of $u$ whose trajectories in $H$ are almost surely $\widetilde{\gamma}$-Hölder with respect to time, for any $\widetilde{\gamma}<\min (1-s,(1-\alpha) / 2-\beta)$.

Proof. The proof is a straightforward application of the Kolmogorov criterion (see for example Th. 3.3, p. 73 in $[8])$.

\subsection{Proof of Theorem $\mathbf{3 . 2}$}

According to (3.9), $u^{n}$ can be written as

$$
\begin{aligned}
u^{n}= & S_{\tau}^{n} u_{0}-\tau \sum_{k=0}^{n-1} S_{\tau}^{n-k-1}(I+\tau \theta A)^{-1} f\left(u^{k}\right) \\
& +\sqrt{\tau} \sum_{k=0}^{n-1} S_{\tau}^{n-k-1}(I+\tau \theta A)^{-1} \sigma\left(u^{k}\right) \chi^{k}
\end{aligned}
$$


where we have set

$$
S_{\tau}=(I+\tau \theta A)^{-1}(I-\tau(1-\theta) A) .
$$

The solution $u$ of (3.1)-(3.2) satisfies the following mild formulation

$$
u(n \tau)=e^{-n \tau A} u_{0}-\int_{0}^{n \tau} e^{-(n \tau-s) A} f(u(s)) \mathrm{d} s+\int_{0}^{n \tau} e^{-(n \tau-s) A} \sigma(u(s)) \mathrm{d} W(s) .
$$

From (3.12) and (3.13), we get

$$
\begin{aligned}
e^{n}= & \left(S_{\tau}-e^{-n \tau A}\right) u_{0} \\
& -\sum_{k=0}^{n-1} \int_{k \tau}^{(k+1) \tau}\left[S_{\tau}^{n-k-1}(I+\tau \theta A)^{-1} f\left(u^{k}\right)-e^{-(n \tau-s) A} f(u(s))\right] \mathrm{d} s \\
& -\sum_{k=0}^{n-1} \int_{k \tau}^{(k+1) \tau}\left[S_{\tau}^{n-k-1}(I+\tau \theta A)^{-1} \sigma\left(u^{k}\right)-e^{-(n \tau-s) A} \sigma(u(s))\right] \mathrm{d} W(s) \\
= & \mathbb{A}+\mathbb{B}+\mathbb{C} .
\end{aligned}
$$

The first term of (3.14) can be easily estimated owing to the following lemma (cf. Th. 1.1, p. 921 in [17]):

Lemma 3.6. Assuming that (3.10) holds, then there exists a constant $C(\theta)>0$, such that

$$
\left|S_{\tau}^{n}-e^{-n \tau A}\right|_{\mathcal{L}(H)} \leq \frac{C(\theta)}{n}, \quad \forall n \geq 1
$$

Owing to the previous lemma, for any $p \geq 2$, we have

$$
|\mathbb{A}|_{L^{p}(\Omega, H)} \leq \frac{C_{1}}{n}\left|u_{0}\right|_{L^{p}(\Omega, H)}
$$

We now treat the second term of (3.14). It is further splitted into three parts

$$
\begin{aligned}
\mathbb{B}= & -\sum_{k=0}^{n-1} \int_{k \tau}^{(k+1) \tau} S_{\tau}^{n-k-1}(I+\tau \theta A)^{-1}\left[f\left(u^{k}\right)-f(u(k \tau))\right] \mathrm{d} s \\
& -\sum_{k=0}^{n-1} \int_{k \tau}^{(k+1) \tau} S_{\tau}^{n-k-1}(I+\tau \theta A)^{-1}[f(u(k \tau))-f(u(s))] \mathrm{d} s \\
& -\sum_{k=0}^{n-1} \int_{k \tau}^{(k+1) \tau}\left[S_{\tau}^{n-k-1}(I+\tau \theta A)^{-1}-e^{-(n \tau-s) A}\right] f(u(s)) \mathrm{d} s \\
= & \mathbb{B}_{1}+\mathbb{B}_{2}+\mathbb{B}_{3} .
\end{aligned}
$$

According to (3.4), we get for any $p \geq 2$,

$$
\begin{aligned}
\left|\mathbb{B}_{1}\right| \leq & L_{f} \sum_{k=0}^{n-1} \tau\left|A^{s} S_{\tau}^{n-k-1}(I+\tau \theta A)^{-1}\right|_{\mathcal{L}(H)}\left|e^{k}\right| \\
\leq & L_{f} \sum_{k=0}^{n-1} \tau\left|A^{s}\left(S_{\tau}^{n-k-1}-e^{-(n-k-1) \tau A}\right)(I+\tau \theta A)^{-1}\right|_{\mathcal{L}(H)}\left|e^{k}\right| \\
& +L_{f} \sum_{k=0}^{n-1} \tau\left|A^{s} e^{-(n-k-1) \tau A}(I+\tau \theta A)^{-1}\right|_{\mathcal{L}(H)}\left|e^{k}\right| .
\end{aligned}
$$


Then, Lemma 3.6 and estimate (2.5) leads to, for any $p \geq 2$,

$$
\mathbb{E}\left|\mathbb{B}_{1}\right|^{p} \leq C_{1}\left(\sum_{k=0}^{n-1} \frac{\tau}{((n-k-1) \tau)^{s}}\left(\mathbb{E}\left|e^{k}\right|^{p}\right)^{1 / p}\right)^{p}
$$

where we have used the Minkowski inequality in the last step.

Using Proposition 3.4, the same computations leads to, for any $p \geq 2$ :

$$
\mathbb{E}\left|\mathbb{B}_{2}\right|^{p} \leq L_{f}^{p}\left(1+\sup _{t \in[0, T]} \mathbb{E}|u(t)|^{p}\right)\left(\sum_{k=0}^{n-1} \frac{\tau}{((n-k-1) \tau)^{s}}\right)^{p} \tau^{\tilde{\gamma} p} .
$$

Since $s<1$, the last sum can be estimated as follows:

$$
\begin{aligned}
\sum_{k=0}^{n-1} \frac{\tau}{((n-k-1) \tau)^{s}} & \leq \sum_{k=0}^{n-1} \int_{k \tau}^{(k+1) \tau} \frac{\mathrm{d} t}{t^{s}} \\
& \leq \frac{1}{1-s} T^{1-s} .
\end{aligned}
$$

Eventually, thanks to estimate (3.8), we get

$$
\mathbb{E}\left|\mathbb{B}_{2}\right|^{p} \leq c_{2}\left(p, \mathbb{E}\left|u_{0}\right|^{p}, T\right) \tau^{\tilde{\gamma} p} .
$$

The term $\mathbb{B}_{3}$ is estimated using (3.5) first

$$
\begin{aligned}
\left|\mathbb{B}_{3}\right| \leq & L_{f} \sum_{k=0}^{n-1} \int_{k \tau}^{(k+1) \tau}\left|A^{s}\left(S_{\tau}^{n-k-1}(I+\tau \theta A)^{-1}-e^{-(n \tau-s) A}\right)\right|_{\mathcal{L}(H)}(1+|u(s)|) \mathrm{d} s \\
\leq & L_{f} \sum_{k=0}^{n-1} \int_{k \tau}^{(k+1) \tau}\left|A^{s}\left(S_{\tau}^{n-k-1}-e^{-(n-k-1) \tau A}\right)(I+\tau \theta A)^{-1}\right|_{\mathcal{L}(H)}(1+|u(s)|) \mathrm{d} s \\
& +L_{f} \sum_{k=0}^{n-1} \int_{k \tau}^{(k+1) \tau}\left|A^{s}\left(e^{-(n-k-1) \tau A}-e^{-(n \tau-s) A}\right)(I+\tau \theta A)^{-1}\right|_{\mathcal{L}(H)}(1+|u(s)|) \mathrm{d} s \\
& +L_{f} \sum_{k=0}^{n-1} \int_{k \tau}^{(k+1) \tau}\left|A^{s} e^{-(n \tau-s) A}\left((I+\tau \theta A)^{-1}-I\right)\right|_{\mathcal{L}(H)}(1+|u(s)|) \mathrm{d} s .
\end{aligned}
$$

Let us estimate the different $\mathcal{L}(H)$-norms which appear in $(3.20)$. Thanks to Lemma 3.6, the $\mathcal{L}(H)$-norm of the first term of the right hand side of (3.20) can be majorized by

$$
\begin{aligned}
\left|A^{s}\left(S_{\tau}^{n-k-1}-e^{-(n-k-1) \tau A}\right)(I+\tau \theta A)^{-1}\right|_{\mathcal{L}(H)} & \leq \frac{C(\theta) \tau^{-s}}{n-k-1} \sup _{i \geq 1}\left(\frac{\left(\lambda_{i} \tau\right)^{s}}{1+\tau \theta \lambda_{i}}\right) \\
& \leq \frac{C(\theta) \tau^{\gamma_{1}}}{((n-k-1) \tau)^{s+\gamma_{1}}}
\end{aligned}
$$

where $\gamma_{1}>0$ is such that $\widetilde{\gamma}<\gamma_{1}<1-s$ which is possible since $s<1$ and $\widetilde{\gamma}<1-s$. 
The following one can be estimated as follows

$$
\begin{aligned}
\left|A^{s}\left(e^{-(n-k-1) \tau A}-e^{-(n \tau-s) A}\right)(I+\tau \theta A)^{-1}\right|_{\mathcal{L}(H)} & \leq \sup _{i \geq 1}\left\{\left(\frac{1-e^{-((k+1) \tau-s) \lambda_{i}}}{1+\tau \theta \lambda_{i}}\right) \lambda_{i}^{s} e^{-(n-k-1) \tau \lambda_{i}}\right\} \\
& \leq \sup _{i \geq 1}\left\{\lambda_{i}^{s} e^{-(n-k-1) \tau \lambda_{i}}\right\} \\
& \leq \frac{C(s) \tau^{\gamma_{1}}}{((n-k-1) \tau)^{s+\gamma_{1}}}
\end{aligned}
$$

where we have used $(2.5)$ in the second line.

Finally, the third $\mathcal{L}(H)$-norm of (3.20) is estimated owing to (2.5) which gives

$$
\begin{aligned}
\left|A^{s} e^{-(n \tau-s) A}\left((I+\tau \theta A)^{-1}-I\right)\right|_{\mathcal{L}(H)} & \leq \sup _{i \geq 1}\left\{\lambda_{i}^{s} e^{-(n \tau-s) \lambda_{i}}\left(\frac{\tau \theta \lambda_{i}}{1+\tau \theta \lambda_{i}}\right)\right\} \\
& \leq \sup _{i \geq 1}\left\{\lambda_{i}^{s} e^{-(n-k-1) \tau \lambda_{i}}\right\} \\
& \leq \frac{C(s) \tau^{\gamma_{1}}}{((n-k-1) \tau)^{s+\gamma_{1}}}
\end{aligned}
$$

since $s \in] k \tau,(k+1) \tau[$.

We take now the expectation of (3.20) to the power $p \geq 2$, and we get, after substuting the last three estimates into the new estimate in $L^{p}(\Omega, H)$ :

$$
\mathbb{E}\left|\mathbb{B}_{3}\right|^{p} \leq C(p, \theta, T, f, s) \tau^{\gamma_{1} p}\left(1+\mathbb{E}\left|u_{0}\right|^{p}\right)
$$

where the sum in $k$ was estimated as in (3.18) since $s+\gamma_{1}<1$. Eventually, (3.17), (3.19) and (3.21) lead to

$$
|\mathbb{B}|_{L^{p}(\Omega, H)} \leq C_{2}\left(\tau^{\tilde{\gamma}}+\sum_{k=0}^{n-1} \frac{\tau}{((n-k-1) \tau)^{s}}\left|e^{k}\right|_{L^{p}(\Omega, H)}\right)
$$

for $\tau \leq 1$ since $\gamma_{1}>\widetilde{\gamma}$.

At last, the stochastic term in (3.14) can be estimated in $L^{p}(\Omega, H)$ using Burkholder-Davis-Gundy inequality:

$$
\mathbb{E}|\mathbb{C}|^{p} \leq C_{p}\left(\sum_{k=0}^{n-1} \int_{k \tau}^{(k+1) \tau}\left(\mathbb{E}\left|\mathbb{D}_{k}(s)\right|_{L_{2}^{0}}^{p}\right)^{2 / p} \mathrm{~d} s\right)^{p / 2}
$$

where we have written

$$
\mathbb{D}_{k}(s)=S_{\tau}^{n-k-1}(I+\tau \theta A)^{-1} \sigma\left(u^{k}\right)-e^{-(n \tau-s) A} \sigma(u(s)) .
$$

Using the same ideas as in (3.16), this last term can be split into three terms:

$$
\mathbb{D}_{k}(s)=\mathbb{D}_{k}^{1}(s)+\mathbb{D}_{k}^{2}(s)+\mathbb{D}_{k}^{3}(s)
$$

where for any $k$ and $s$ in $] k \tau,(k+1) \tau[$,

$$
\begin{aligned}
& \mathbb{D}_{k}^{1}(s)=S_{\tau}^{n-k-1}(I+\tau \theta A)^{-1}\left[\sigma\left(u^{k}\right)-\sigma(u(k \tau))\right], \\
& \mathbb{D}_{k}^{2}(s)=S_{\tau}^{n-k-1}(I+\tau \theta A)^{-1}[\sigma(u(k \tau))-\sigma(u(s))], \\
& \mathbb{D}_{k}^{3}(s)=\left[S_{\tau}^{n-k-1}(I+\tau \theta A)^{-1}-e^{-(n \tau-s) A}\right] \sigma(u(s)) .
\end{aligned}
$$


Using (3.6), taking the $L_{2}^{0}$-norm of $\mathbb{D}_{k}^{1}(s)$ leads to

$$
\begin{aligned}
\left(\mathbb{E}\left|\mathbb{D}_{k}^{1}(s)\right|_{L_{2}^{0}}^{p}\right)^{2 / p} \leq & L_{\sigma}^{2}\left(2\left|A^{\beta}\left(S_{\tau}^{n-k-1}-e^{-(n-k-1) \tau A}\right)(I+\tau \theta A)^{-1}\right|_{L_{2}^{0}}^{2}\right. \\
& \left.+2\left|A^{\beta} e^{-(n-k-1) \tau A}(I+\tau \theta A)^{-1}\right|_{L_{2}^{0}}^{2}\right)\left(\mathbb{E}\left|e^{k}\right|^{p}\right)^{2 / p} \\
\leq & (a+b)\left(\mathbb{E}\left|e^{k}\right|^{p}\right)^{2 / p} .
\end{aligned}
$$

Then, using Lemma 3.6, we have for $\gamma \in(0,1)$,

$$
\begin{aligned}
a & \leq \frac{C(\theta)}{(n-k-1)^{2}} \sum_{i \geq 1} \frac{\lambda_{i}^{2 \beta}}{\left(1+\tau \theta \lambda_{i}\right)^{2}} \\
& \leq \frac{C(\theta)}{(n-k-1)^{2}}\left(\sum_{i \geq 1} \lambda_{i}^{2 \beta+\gamma-1}\right) \sup _{i \geq 1}\left\{\frac{\lambda_{i}^{1-\gamma}}{\left(1+\tau \theta \lambda_{i}\right)^{2}}\right\} \\
& \leq \frac{C(\theta)}{(n-k-1)^{1-\gamma}}\left(\sum_{i \geq 1} \lambda_{i}^{2 \beta+\gamma-1}\right) \sup _{i \geq 1}\left\{\frac{\left(\lambda_{i} \tau\right)^{1-\gamma}}{\left(1+\tau \theta \lambda_{i}\right)^{1-\gamma}}\right\} \tau^{\gamma-1},
\end{aligned}
$$

since $1+\tau \theta \lambda_{i} \geq 1$ so that $\left(1+\tau \theta \lambda_{i}\right)^{2} \geq\left(1+\tau \theta \lambda_{i}\right)^{1-\gamma}$. In the same way, we have used $(n-k-1)^{2} \geq(n-k-1)^{1-\gamma}$ for every $k=0, \ldots, n-1$. Finally with $\gamma$ chosen such that (3.3) holds, we get

$$
a \leq \frac{C(\theta)}{((n-k-1) \tau)^{1-\gamma}} .
$$

Next, (2.5) and (3.3) allow us to estimate easily $b$ as

$$
b \leq \frac{C^{\prime}}{((n-k-1) \tau)^{1-\gamma}} .
$$

Substituting the estimate for $a$ and $b$ in (3.25) yields

$$
\left(\mathbb{E}\left|\mathbb{D}_{k}^{1}(s)\right|^{p}\right)^{2 / p} \leq \frac{C^{\prime \prime}(\theta)}{((n-k-1) \tau)^{1-\gamma}}\left(\mathbb{E}\left|e^{k}\right|^{p}\right)^{2 / p} .
$$

for $\gamma>0$ such that (3.3) holds.

Next, Proposition 3.4, estimate (3.8) and similar computations lead to

$$
\left(\mathbb{E}\left|\mathbb{D}_{k}^{2}(s)\right|^{p}\right)^{2 / p} \leq C\left(p, \sigma, \theta, T,\left|u_{0}\right|_{L^{p}(\Omega, H)}\right) \frac{\tau^{2 \tilde{\gamma}}}{((n-k-1) \tau)^{1-\gamma}} .
$$

Finally, using first $(3.7),\left(\mathbb{E}\left|\mathbb{D}_{k}^{3}(s)\right|^{p}\right)^{2 / p}$ can be estimated as follows:

$$
\begin{aligned}
\left(\mathbb{E}\left|\mathbb{D}_{k}^{3}(s)\right|^{p}\right)^{2 / p} \leq & L_{\sigma}^{2}\left|A^{\beta}\left(S_{\tau}^{n-k-1}-e^{-(n-k-1) \tau A}\right)(I+\tau \theta A)^{-1}\right|_{L_{2}^{0}}^{2}\left(1+\left(\mathbb{E}|u(s)|^{p}\right)^{2 / p}\right) \\
& +L_{\sigma}^{2}\left|A^{\beta}\left(e^{-(n-k-1) \tau A}-e^{-(n \tau-s) A}\right)(I+\tau \theta A)^{-1}\right|_{L_{2}^{0}}^{2}\left(1+\left(\mathbb{E}|u(s)|^{p}\right)^{2 / p}\right) \\
& +L_{\sigma}^{2}\left|A^{\beta} e^{-(n \tau-s) A}\left((I+\tau \theta A)^{-1}-I\right)\right|_{L_{2}^{0}}^{2}\left(1+\left(\mathbb{E}|u(s)|^{p}\right)^{2 / p}\right) .
\end{aligned}
$$


The first $L_{2}^{0}$-norm in the right hand side of (3.28) has already been estimated ( $c f . a$ in (3.25)), so we get

$$
\left|A^{\beta}\left(S_{\tau}^{n-k-1}-e^{-(n-k-1) \tau A}\right)(I+\tau \theta A)^{-1}\right|_{L_{2}^{0}}^{2} \leq \frac{C(\theta) \tau^{\gamma_{2}}}{((n-k-1) \tau)^{1-\gamma+\gamma_{2}}}
$$

where $\gamma_{2}>0$ is such that $2 \widetilde{\gamma}<\gamma_{2}<\gamma$, which is possible since $\gamma>2 \widetilde{\gamma}$.

The second one can be majorized as follows

$$
\begin{aligned}
\left|A^{\beta}\left(e^{-(n-k-1) \tau A}-e^{-(n \tau-s) A}\right)(I+\tau \theta A)^{-1}\right|_{L_{2}^{0}}^{2} & \leq C \sup _{i \geq 1}\left\{\frac{\left(1-e^{-((k+1) \tau-s) \lambda_{i}}\right)^{2}}{\left(1+\tau \theta \lambda_{i}\right)^{2}} \lambda_{i}^{1-\gamma} e^{-2(n-k-1) \tau \lambda_{i}}\right\} \\
& \leq C \sup _{i \geq 1}\left\{\left(\tau \lambda_{i}\right)^{\gamma_{2}} \lambda_{i}^{1-\gamma} e^{-2(n-k-1) \tau \lambda_{i}}\right\} \\
& \leq C \tau^{\gamma_{2}} \sup _{i \geq 1}\left\{\lambda_{i}^{1-\gamma+\gamma_{2}} e^{-2(n-k-1) \tau \lambda_{i}}\right\} \\
& \leq \frac{C(\gamma) \tau^{\gamma_{2}}}{((n-k-1) \tau)^{1-\gamma+\gamma_{2}}}
\end{aligned}
$$

where we have used (3.3) in the first line. In the second line, we have used the fact that for any $\delta$ in $[0,1]$ (here $\delta=\gamma_{2}$ ), there exists a constant $c_{\delta}>0$ such that $\left|e^{-x}-e^{-y}\right| \leq c_{\delta}|x-y|^{\delta}$, for all $x, y \geq 0(c f$. also (2.6)). Finally, (2.5) yields the last line.

Eventually, (2.5) and (3.3) lead to

$$
\left|A^{\beta} e^{-(n \tau-s) A}\left((I+\tau \theta A)^{-1}-I\right)\right|_{L_{2}^{0}}^{2} \leq \frac{C(\gamma) \tau^{\gamma_{2}}}{((n-k-1) \tau)^{1-\gamma+\gamma_{2}}} .
$$

After substituting the last three estimates in (3.28) and using the estimate (3.8), we get

$$
\left(\mathbb{E}\left|\mathbb{D}_{k}^{3}(s)\right|^{p}\right)^{2 / p} \leq C\left(\gamma, \theta,\left|u_{0}\right|_{L^{p}(\Omega, H)}, \sigma\right) \frac{\tau^{2 \bar{\gamma}}}{((n-k-1) \tau)^{1-\gamma+\gamma_{2}}},
$$

for $\tau \leq 1$, since $\gamma_{2}>2 \widetilde{\gamma}$.

At last, (3.26), (3.27) and (3.19) can be put back together into (3.23) in order to obtain

$$
|\mathbb{C}|_{L^{p}(\Omega)} \leq C_{3}\left(\tau^{\tilde{\gamma}}+\left(\sum_{k=0}^{n-1} \frac{\tau}{((n-k-1) \tau)^{1-\gamma}}\left|e^{k}\right|_{L^{p}(\Omega, H)}^{2}\right)^{1 / 2}\right),
$$

where $C_{3}=C\left(p, \gamma, \theta,\left|u_{0}\right|_{L^{p}(\Omega, H)}, \sigma, T\right)$. Let us note that again we have used (3.18) in order to estimate the remaining sum in $k$ since $1-\gamma+\gamma_{2}<1$.

Lastly, we can estimate $\left|e^{n}\right|_{L^{p}(\Omega, H)}$ by gathering (3.15), (3.22) and (3.30) and this yields

$$
\begin{aligned}
\left|e^{n}\right|_{L^{p}(\Omega, H)} \leq & C_{4}\left(\tau^{\bar{\gamma}}+\frac{1}{n}\left|u_{0}\right|_{L^{p}(\Omega, H)}+\sum_{k=0}^{n-1} \frac{\tau}{((n-k-1) \tau)^{s}}\left|e^{k}\right|_{L^{p}(\Omega, H)}\right. \\
& +\left(\sum_{k=0}^{n-1} \frac{\tau}{((n-k-1) \tau)^{1-\gamma}}\left|e^{k}\right|_{L^{p}(\Omega, H)}^{2}\right)
\end{aligned}
$$


We now take an integer $q \geq 1$ such that $\frac{1}{2 q} \leq \widetilde{\gamma}$ and estimate $\left|e^{n}\right|_{L^{p}(\Omega, H)}^{2 q}$. First, the Hölder inequality in the first sum in (3.31) leads to

$$
\begin{aligned}
\sum_{k=0}^{n-1} \frac{\tau}{((n-k-1) \tau)^{s}}\left|e^{k}\right|_{L^{p}(\Omega, H)} & \leq\left(\sum_{k=0}^{n-1} \frac{\tau}{((n-k-1) \tau)^{s} \frac{2 q}{2 q-1}}\right)^{\frac{2 q-1}{2 q}}\left(\sum_{k=0}^{n-1} \tau\left|e^{k}\right|_{L^{p}(\Omega, H)}^{2 q}\right)^{\frac{1}{2 q}} \\
& \leq \frac{1}{1-s \frac{2 q}{2 q-1}} T^{\frac{2 q-1}{2 q}-s}\left(\sum_{k=0}^{n-1} \tau\left|e^{k}\right|_{L^{p}(\Omega, H)}^{2 q}\right)^{\frac{1}{2 q}}
\end{aligned}
$$

where we have used (3.18) in the second line, since $1 / 2 q \leq \widetilde{\gamma}<1-s$.

Next, the second sum is majorized as

$$
\begin{aligned}
\left(\sum_{k=0}^{n-1} \frac{\tau}{((n-k-1) \tau)^{1-\gamma}}\left|e^{k}\right|_{L^{p}(\Omega, H)}^{2}\right)^{1 / 2} & \leq\left(\sum_{k=0}^{n-1} \frac{\tau}{((n-k-1) \tau)^{(1-\gamma) \frac{q}{q-1}}}\right)^{\frac{q-1}{2 q}}\left(\sum_{k=0}^{n-1} \tau\left|e^{k}\right|_{L^{p}(\Omega, H)}^{2 q}\right)^{\frac{1}{2 q}} \\
& \leq \frac{1}{1-(1-\gamma) \frac{q}{q-1}} T^{\left(\gamma-\frac{1}{q}\right) / 2}\left(\sum_{k=0}^{n-1} \tau\left|e^{k}\right|_{L^{p}(\Omega, H)}^{2 q}\right)^{\frac{1}{2 q}}
\end{aligned}
$$

where we have used again (3.18) since $1 / 2 q \leq \widetilde{\gamma}<\gamma / 2$. Eventually, taking the power $2 q$ of (3.31) yields

$$
\left|e^{n}\right|_{L^{p}(\Omega, H)}^{2 q} \leq C_{5}(q, T)\left(\left(\tau^{\tilde{\gamma}}+\frac{1}{n}\left|u_{0}\right|_{L^{p}(\Omega, H)}\right)^{2 q}+\sum_{k=0}^{n-1} \tau\left|e^{k}\right|_{L^{p}(\Omega, H)}^{2 q}\right)
$$

Applying the discrete Gronwall Lemma 2.3 with $\xi_{n}=\left|e^{n}\right|_{L^{p}(\Omega, H)}^{2 q}, \eta_{n}=\left(\tau^{\bar{\gamma}}+\frac{1}{n}\left|u_{0}\right|_{L^{p}(\Omega, H)}\right)^{2 q}$ and $L=$ $C_{5}(q, T) \tau$, yields the result. Namely according to Remark 2.4,

$$
\left|e^{n}\right|_{L^{p}(\Omega, H)} \leq C_{6}(q, T)\left(\tau^{\tilde{\gamma}}+\frac{1}{n}\left|u_{0}\right|_{L^{p}(\Omega, H)}\right)
$$

for any $0 \leq n \leq N$. This ends the proof of Theorem 3.2.

\section{The LOCAlly Lipschitz CASE}

In this section, we deal with a non linearity $f$ which is only locally Lipschitz. For the sake of clarity, we will treat this case in a one-dimensional framework and

$$
f(u)=\frac{\partial}{\partial x}\left(\frac{u^{2}}{2}\right)
$$

but the ideas we introduce are general and can be applied in more general situations.

We first study the following continuous Cauchy Problem: find a stochastic process $u$ such that

$$
\mathrm{d} u+(A u+f(u)) \mathrm{d} t=\sigma(u) \mathrm{d} W
$$

with the initial condition

$$
u(0)=u_{0}
$$


Here, $A$ denotes the operator $-\frac{\partial^{2}}{\partial x^{2}}$ on $(0,1)$ with either Dirichlet or Neumann boundary conditions. We know that $A$ is an unbounded self-adjoint operator on $H=L^{2}(0,1)$, whose domain $D(A)$ is compactly embedded in $H$. We denote in the same way as in the previous section by $\left\{\lambda_{i}\right\}_{i \geq 1}$ its eigenvalues and by $\left\{e_{i}\right\}_{i \geq 1}$ its eigenfunctions. In the case of Burgers equation, we have

$$
e_{i}(x)=\sqrt{\frac{2}{\pi}} \sin (i \pi x), \quad x \in(0,1),
$$

and

$$
\lambda_{i}=i^{2} \pi^{2}
$$

Let $\alpha$ be such that $\sum_{i \geq 1} \lambda_{i}^{-\alpha}<+\infty$. Then, we know that here we can take any

$$
\alpha>\frac{1}{2}
$$

The assumptions concerning the noise is strengthened; $\{W(t)\}_{t \in[0, T]}$ remains the same as in Section 3, i.e. a cylindrical Wiener process on $L^{2}(0,1)$ but here $\sigma$ is Lipschitz continuous from $L^{2}(0,1)$ into $\mathcal{L}\left(L^{2}(0,1), D\left(A^{-\beta}\right)\right)$ with

$$
\beta=0
$$

and there exists a constant $L_{\sigma}>0$ such that

$$
|\sigma(u)-\sigma(v)|_{\mathcal{L}\left(L^{2}(0,1)\right)} \leq L_{\sigma}|u-v|
$$

Moreover, we take the extra assumption

$$
\forall u \in L^{2}(0,1), \quad|\sigma(u)|_{\mathcal{L}\left(L^{2}(0,1)\right)} \leq C_{\sigma},
$$

for some constant $C_{\sigma}>0$.

Remark 4.1. For example, we can consider the case where, $\sigma: \mathbb{R} \rightarrow \mathbb{R}$ is a bounded function and $\sigma(u)$ is the pointwise multiplication by $\sigma(u)$, which, with abusive notation, can be denoted again by $\sigma(u)$.

The results concerning this problem can be summarized in the following proposition (see [7]).

Proposition 4.2. Let $T>0$. Let $p$ be an integer such that $p \geq 2$ and $u_{0} \in L^{p}(\Omega, H)$. Then, under the assumptions (4.1), (4.6) and (4.7), there exists a unique stochastic process u solution to the Cauchy Problem (4.2)-(4.3). Moreover, we have

$$
\lim _{M \rightarrow+\infty} \mathbb{P}\left\{\sup _{t \in[0, T]}|u(t)| \geq M\right\}=0 .
$$

For any positive real $T$ and positive integer $N$, we set

$$
\tau=\frac{T}{N}
$$

and we denote by $\left\{u^{n}\right\}_{0 \leq n \leq N}$ the sequence in $H$ defined by the following induction

$$
\left\{\begin{array}{l}
u^{n+1}-u^{n}+\tau\left(A u^{n+\theta}+f\left(u^{n}\right)\right)=\sqrt{\tau} \sigma\left(u^{n}\right) \chi^{n}, \\
u^{0}=u_{0}
\end{array}\right.
$$

where the notations are the same as in Section 3. 
We eventually set for any $n$ in $\{0, \ldots, N\}$,

$$
e_{n}=u^{n}-u(n \tau),
$$

where $u$ is the solution given by Proposition 4.2.

The main result of this section is:

Theorem 4.3. Let $T>0$ and $u_{0} \in L^{p}\left(\Omega, L^{2}(0,1)\right)$ for some integer $p \geq 2$. Let $u$ be the solution of (4.2)-(4.3) on $[0, T]$ given by Proposition 4.2, and $\left\{u^{n}\right\}_{n \geq 0}$ defined by the numerical scheme (4.8).

Then, scheme (4.8) is convergent in probability to $u$. Moreover, for any $\widetilde{\gamma}<1 / 4$, scheme (4.8) is of order in probability $\widetilde{\gamma}$ in $L^{2}(0,1)$. Indeed, we have

$$
\lim _{C \rightarrow+\infty} \limsup _{\tau \rightarrow 0} \mathbb{P}\left\{\max _{1 \leq n \tau \leq T}\left|e_{n}\right| \geq C\left(\tau^{\bar{\gamma}}+\tau\left(\mathbb{E}\left|u_{0}\right|^{p}\right)^{1 / p}\right)\right\}=0,
$$

for any $\widetilde{\gamma}<1 / 4$.

The proof of Proposition 4.2 can be found in [7]. We will give here in the following subsection the outline of this proof. First it consists in truncating the nonlinearity $f$ in order to recover a Lipschitz function. Then the truncation is removed according to an a priori estimate of the solution of the truncated equation uniformly with respect to the parameter of truncation.

Theorem 4.3 will be proved in the last subsection. It consists first in proving that the scheme converges in probability. This implies boundedness in probability of the approximating sequence $\left\{u^{n}\right\}$ and allows us to use the results in the globally Lipschitz case.

\subsection{Outline of the proof of Proposition 4.2}

Let $\left.\varphi \in \mathcal{C}_{0}^{\infty}(\mathbb{R}), \operatorname{supp}(\varphi) \subset\right]-2,+2[, \varphi(x)=1$, if $|x| \leq 1$. For any $R>0$, we put

$$
\varphi_{R}(x)=\varphi\left(\frac{x}{R}\right),
$$

and

$$
f_{R}(u)=\varphi_{R}(|u|) f(u) .
$$

Then, we have the following lemma whose proof is left to the reader.

Lemma 4.4. For any $s>3 / 4$ and $R>0$, there exists some constants $C_{1}(s), C_{2}(s, R)>0$ such that for any $(u, v)$ in $L^{2}(0,1)$, one has

$$
\begin{aligned}
|f(u)-f(v)|_{D\left(A^{-s}\right)} & \leq C_{1}(s)(|u|+|v|)|u-v|, \\
\left|f_{R}(u)-f_{R}(v)\right|_{D\left(A^{-s}\right)} & \leq C_{2}(s, R)|u-v| .
\end{aligned}
$$

Let the following Cauchy Problem: find $u_{R}, R>0$, such that

$$
\mathrm{d} u_{R}+\left(A u_{R}+f_{R}\left(u_{R}\right)\right) \mathrm{d} t=\sigma\left(u_{R}\right) \mathrm{d} W,
$$

with the initial condition

$$
u_{R}(0)=u_{0}
$$

Owing to (4.13), (4.6) and Proposition 3.1, we know that for any $R>0$ and for any $T>0$, there exists a unique stochastic process $u_{R}$ solution of (4.14)-(4.15). The proof of Proposition 4.2 essentially consists in deriving 
a priori estimate on $u_{R}$ in $L^{2}(\Omega, \mathcal{C}([0, T], H))$ uniformly with respect to $R$. The first step consists in deriving similar estimate on the solution of the pseudo-linear problem

$$
\left\{\begin{array}{l}
\mathrm{d} z_{R}+A z_{R} \mathrm{~d} t=\sigma\left(u_{R}\right) \mathrm{d} W \\
z_{R}(0)=0
\end{array}\right.
$$

whose solution is given by

$$
z_{R}(t)=\int_{0}^{t} e^{-(t-\tau) A} \sigma\left(u_{R}(\tau)\right) \mathrm{d} W(\tau) .
$$

Those estimates are given in the following Proposition (see [7]):

Proposition 4.5. For any $T>0$, there exists some constants $C_{i}(T)>0, i=1,2$, such that

$$
\begin{gathered}
\mathbb{E} \sup _{t \in[0, T]}\left|z_{R}(t)\right| \leq C_{1}(T), \\
\mathbb{E} \sup _{t \in[0, T]}\left|z_{R}(t)\right|_{L^{4}(0,1)}^{4} \leq C_{2}(T),
\end{gathered}
$$

where $C_{i}(T), i=1,2$, are locally bounded functions of $T$.

Now Proposition 4.5 implies the following a priori estimate on the solution $u_{R}$ of the Cauchy Problem (4.2)(4.3) (see again [7]):

Proposition 4.6. For any $T>0$,

$$
\lim _{M \rightarrow+\infty} \limsup _{R \rightarrow+\infty} \mathbb{P}\left\{\sup _{t \in[0, T]}\left|u_{R}(t)\right| \geq M\right\}=0 .
$$

Finally, existence and uniqueness of a stochastic process solution of (4.2)-(4.3) will be deduced from Propositions 4.5 and 4.6. Such computations can be summarized in the following lemma (see [7]).

Lemma 4.7. Let $R>0$ and

$$
\tau_{R}=\inf \left\{t \in[0, T]|\quad| u_{R}(t) \mid \geq R\right\} \wedge T
$$

Then we have

- $\left\{\tau_{R}\right\}_{R>0}$ is non-decreasing with respect to $R$,

- $\lim _{R \rightarrow+\infty} \tau_{R}=T$, a.s.,

- $\forall t \leq \tau_{R}, \quad u_{R}(t)=u(t)$, a.s., where $u$ satisfies the mild formulation, a.s.,

$$
u(t)=e^{-t A} u_{0}-\int_{0}^{t} e^{-(t-\tau) A} \frac{\partial}{\partial x}\left(\frac{u^{2}}{2}\right) \mathrm{d} \tau+\int_{0}^{t} e^{-(t-\tau) A} \sigma(u(\tau)) \mathrm{d} W(\tau) .
$$

\subsection{Proof of Theorem $\mathbf{4 . 3}$}

Similarly as in the begining of this section, we define $\left\{u_{R}^{n}\right\}_{0 \leq n \leq N}$ for any $R>0$, by the induction

$$
\left\{\begin{array}{l}
u_{R}^{n+1}-u_{R}^{n}+\tau\left(A u_{R}^{n+\theta}+f_{R}\left(u_{R}^{n}\right)\right)=\sqrt{\tau} \sigma\left(u_{R}^{n}\right) \chi^{n} \\
u_{R}^{0}=u_{0}
\end{array}\right.
$$


and we set for any $n$ in $\{0, \ldots, N\}$,

$$
e_{R}^{n}=u_{R}^{n}-u_{R}(n \tau),
$$

where the notations are the same as in the previous subsections.

Let $u$ and $u^{n}$ be given respectively by Proposition 4.2 and Scheme (4.8). The proof of Theorem 4.3 relies mainly on the following Lemma:

Lemma 4.8. Scheme (4.8) is convergent in probability to $u$. More precisely, for any $\varepsilon$ in $(0,1)$ and $R>0$, we have

$$
\lim _{N \rightarrow+\infty} \mathbb{P}\left\{\max _{0 \leq n \leq N}\left|e^{n}\right| \geq \varepsilon\right\}=0
$$

and

$$
\lim _{M \rightarrow+\infty} \limsup _{N \rightarrow+\infty} \mathbb{P}\left\{\max _{0 \leq n \leq N}\left|u^{n}\right| \geq M\right\}=0
$$

Proof. For any $\varepsilon$ in $(0,1), R>0$ and any integer $N$, similarly as in [12], we define the following random variables:

$$
\begin{aligned}
& \theta_{R}=\inf \quad\{t \leq T, \quad|u(t)| \geq R-1\} \quad \text { a.s. } \\
& n_{\varepsilon}=\min \quad\left\{n \leq N, \quad\left|u^{n}-u(n \tau)\right| \geq \varepsilon\right\} \quad \text { a.s. }
\end{aligned}
$$

We have the following inclusion

$$
\begin{aligned}
& \left\{\max _{0 \leq n \leq N}\left|e^{n}\right| \geq \varepsilon\right\} \subset\left[\left\{\theta_{R}<T\right\} \cap\left\{\max _{0 \leq n \leq N}\left|e^{n}\right| \geq \varepsilon\right\}\right] \\
& \cup\left[\left\{\theta_{R} \geq T\right\} \cap\left\{n_{\varepsilon} \tau<T\right\} \cap\left\{\max _{0 \leq n \leq N}\left|e^{n}\right| \geq \varepsilon\right\}\right] \\
& \cup\left[\left\{\theta_{R} \geq T\right\} \cap\left\{n_{\varepsilon} \tau \geq T\right\} \cap\left\{\max _{0 \leq n \leq N}\left|e^{n}\right| \geq \varepsilon\right\}\right] .
\end{aligned}
$$

Since the last event is empty, the last inclusion can be reduced to

$$
\left\{\max _{0 \leq n \leq N}\left|e^{n}\right| \geq \varepsilon\right\} \subset\left[\left\{\theta_{R}<T\right\} \cup\left[\left\{\theta_{R} \geq T\right\} \cap\left\{n_{\varepsilon} \tau<T\right\}\right]\right] .
$$

On the one hand, since $n \tau \leq T \leq \theta_{R}$ in the second event of the right hand side of the previous inclusion, we deduce that for any $n \leq N,|u(n \tau)| \leq R-1 \leq R$. Hence thanks to Lemma 4.7, we know that $u(n \tau)=u_{R}(n \tau)$ for any $n \leq N$.

On the other hand, if $n_{\varepsilon}<N$ then $\left|u^{n_{\varepsilon}-1}-u\left(\left(n_{\varepsilon}-1\right) \tau\right)\right|<\varepsilon<1$ and so $\left|u^{n_{\varepsilon}-1}\right| \leq R$. Then we deduce from (4.22) that $u^{n_{\varepsilon}}=u_{R}^{n_{\varepsilon}}$. We can then replace $e^{n_{\varepsilon}}$ by $e_{R}^{n_{\varepsilon}}$. Finally, we conclude to

$$
\mathbb{P}\left\{\max _{0 \leq n \leq N}\left|e^{n}\right| \geq \varepsilon\right\} \leq \mathbb{P}\left\{\theta_{R}<T\right\}+\mathbb{P}\left\{\max _{0 \leq n \leq N}\left|e_{R}^{n}\right| \geq \varepsilon\right\}
$$

Next, thanks to Theorem 3.2, we can take the limit in $N$ in (4.25) by the mean of Bienaymé-Tchebycheff inequality:

$$
\lim _{N \rightarrow+\infty} \mathbb{P}\left\{\max _{0 \leq n \leq N}\left|e^{n}\right| \geq \varepsilon\right\} \leq \mathbb{P}\left\{\theta_{R}<T\right\} .
$$

Then, owing to Lemma 4.7, we can take the limit in $R\left(\tau_{R}\right.$ being replaced by $\left.\theta_{R}\right)$ and we eventually get (4.23). 
Then (4.23) and the result of boundedness in probability of Proposition 4.2 implies that $\left\{u_{n}\right\}_{0 \leq n \leq N}$ is bounded in probability, uniformly with respect to $N$. Indeed, we have for any $M>0$ :

$$
\mathbb{P}\left\{\max _{0 \leq n \leq N}\left|u_{n}\right| \geq M\right\} \leq \mathbb{P}\left\{\max _{0 \leq n \leq N}\left|e_{n}\right| \geq \varepsilon\right\}+\mathbb{P}\left\{\sup _{t \in[0, T]}|u(t)| \geq M-\varepsilon\right\} .
$$

Thus, (4.23) allow us to take the supremum limit in $N$ in the previous inequality for a fixed $M \geq 1$. Then we can take the limit with respect to $M$ and get according to Proposition 4.2 the result (4.24).

Now, we are able to conclude the proof of Theorem 4.3. Let $\widetilde{\gamma}<1 / 4$, then we can write for some fixed $M \geq 1, N \geq 1, \tau>0$ such that $N \tau=T$ and $C>0$,

$$
\begin{aligned}
\mathbb{P}\left\{\max _{1 \leq n \tau \leq T}\left|e^{n}\right| \geq C\left(\tau^{\tilde{\gamma}}+\tau\left(\mathbb{E}\left|u_{0}\right|^{p}\right)^{1 / p}\right)\right\} \leq & \mathbb{P}\left\{\max _{1 \leq n \tau \leq T}\left|e_{M}^{n}\right| \geq C\left(\tau^{\bar{\gamma}}+\tau\left(\mathbb{E}\left|u_{0}\right|^{p}\right)^{1 / p}\right)\right\} \\
& +\mathbb{P}\left\{\max _{0 \leq n \leq N}\left|u_{n}\right| \geq M\right\} \\
& +\mathbb{P}\left\{\sup _{t \in[0, T]}|u(t)| \geq M\right\} .
\end{aligned}
$$

By the mean of the Bienaymé-Tchebycheff inequality and Theorem 3.2, we estimate the first term of the right hand of (4.26). Hence, since $\widetilde{\gamma}<1 / 4,(4.4),(4.5)$ and (4.13) lead to

$$
\begin{aligned}
\mathbb{P}\left\{\max _{1 \leq n \tau \leq T}\left|e^{n}\right| \geq C\left(\tau^{\bar{\gamma}}+\tau\left(\mathbb{E}\left|u_{0}\right|^{p}\right)^{1 / p}\right)\right\} \leq & \frac{C(M, p)}{C^{p}}+\mathbb{P}\left\{\max _{0 \leq n \leq N}\left|u_{n}\right| \geq M\right\} \\
& +\mathbb{P}\left\{\sup _{t \in[0, T]}|u(t)| \geq M\right\}
\end{aligned}
$$

where the constant $C(M, p)$ does not depend on $\tau$ or $N$.

Next, taking the limitsup in $\tau$ towards 0 in (4.27) yields

$$
\begin{aligned}
\limsup _{\tau \rightarrow 0} \mathbb{P}\left\{\max _{1 \leq n \tau \leq T}\left|e^{n}\right| \geq C\left(\tau^{\bar{\gamma}}+\tau\left(\mathbb{E}\left|u_{0}\right|^{p}\right)^{1 / p}\right)\right\} \leq & \frac{C(M, p)}{C^{p}}+\limsup _{\tau \rightarrow 0} \mathbb{P}\left\{\max _{0 \leq n \leq N}\left|u_{n}\right| \geq M\right\} \\
& +\mathbb{P}\left\{\sup _{t \in[0, T]}|u(t)| \geq M\right\}
\end{aligned}
$$

Eventually, we take the limit in $C$. Equation (4.24) and estimate of Proposition 4.2 yield the result (4.9).

\section{Appendix: Proof of Proposition 3.4}

Let $u$ be the solution of (3.1)-(3.2). Then, for any $\left(t_{1}, t_{2}\right)$ in $[0, T], t_{2}<t_{1}$ for example, $u\left(t_{1}\right)-u\left(t_{2}\right)$ can be split into three parts, namely:

$$
\begin{aligned}
u\left(t_{1}\right)-u\left(t_{2}\right)= & \left(e^{-t_{1} A}-e^{-t_{2} A}\right) u_{0} \\
& +\int_{0}^{t_{1}} e^{-\left(t_{1}-s\right) A} f(u(s)) \mathrm{d} s-\int_{0}^{t_{2}} e^{-\left(t_{2}-s\right) A} f(u(s)) \mathrm{d} s \\
& +\int_{0}^{t_{1}} e^{-\left(t_{1}-s\right) A} \sigma(u(s)) \mathrm{d} W(s)-\int_{0}^{t_{2}} e^{-\left(t_{2}-s\right) A} \sigma(u(s)) \mathrm{d} W(s) .
\end{aligned}
$$


Let us estimate the first term in $L^{2}$-norm. We take $\eta \in[0,1]$ and write

$$
\begin{aligned}
\left|\left(e^{-t_{1} A}-e^{-t_{2} A}\right) u_{0}\right| & =\left|\left(e^{-\left(t_{1}-t_{2}\right) A}-I d\right) e^{-t_{2} A} u_{0}\right| \\
& \leq C\left|t_{1}-t_{2}\right|^{\eta} t_{2}^{-\eta}\left|u_{0}\right|,
\end{aligned}
$$

where we have used (2.5) and (2.6) in the right hand side. The second term of the right hand side of (4.29) can be split into two terms:

$$
\begin{array}{rl}
\int_{0}^{t_{1}} e^{-\left(t_{1}-s\right) A} & f(u(s)) \mathrm{d} s-\int_{0}^{t_{2}} e^{-\left(t_{2}-s\right) A} f(u(s)) \mathrm{d} s \\
& =\int_{0}^{t_{2}}\left(e^{-\left(t_{1}-s\right) A}-e^{-\left(t_{2}-s\right) A}\right) f(u(s)) \mathrm{d} s+\int_{t_{2}}^{t_{1}} e^{-\left(t_{1}-s\right) A} f(u(s)) \mathrm{d} s \\
& =\mathrm{I}+\mathbb{I},
\end{array}
$$

where I and II can be estimated as follows. Using (2.5), (2.6) and assumption (3.5):

$$
\begin{aligned}
|\mathrm{I}| & \leq L_{f} \int_{0}^{t_{2}}\left|A^{s}\left(e^{-\left(t_{1}-s\right) A}-e^{-\left(t_{2}-s\right) A}\right)\right|(1+|u(s)|) \mathrm{d} s \\
& \leq C L_{f} \int_{0}^{t_{2}}\left|A^{s+\gamma_{1}} e^{-\left(t_{2}-s\right) A}\right|\left|A^{-\gamma_{1}}\left(e^{-\left(t_{1}-t_{2}\right) A}-I d\right)\right|(1+|u(s)|) \mathrm{d} s \\
& \leq C L_{f}\left|t_{1}-t_{2}\right|^{\gamma_{1}} \int_{0}^{t_{2}} \frac{1}{\left|t_{2}-s\right|^{s+\gamma_{1}}}(1+|u(s)|) \mathrm{d} s,
\end{aligned}
$$

where $\gamma_{1}$ is such that $s+\gamma_{1}<1$.

Then, for any integer $p \geq 1$, we get

$$
\begin{aligned}
\left(\mathbb{E}||^{p}\right)^{1 / p} & \leq C\left|t_{1}-t_{2}\right|^{\gamma_{1}}\left|\int_{0}^{t_{2}} \frac{1}{\left|t_{2}-s\right|^{s+\gamma_{1}}}(1+|u(s)|) \mathrm{d} s\right|_{L^{p}(\Omega)} \\
& \leq C\left|t_{1}-t_{2}\right|^{\gamma_{1}}\left(\sup _{t \in[0, T]}\left(\mathbb{E}|u(t)|^{p}\right)^{1 / p}+1\right) .
\end{aligned}
$$

With the same method, we get for II:

$$
\left(\mathbb{E}|\mathbb{I}|^{p}\right)^{1 / p}|\leq C| t_{1}-\left.t_{2}\right|^{1-s}\left(\sup _{t \in[0, T]}\left(\mathbb{E}|u(t)|^{p}\right)^{1 / p}+1\right) .
$$

In the same manner, the third term of the right hand side of (4.29) can be written as :

$$
\begin{aligned}
\int_{0}^{t_{1}} e^{-\left(t_{1}-s\right) A} \sigma(u(s)) \mathrm{d} W(s)-\int_{0}^{t_{2}} e^{-\left(t_{2}-s\right) A} \sigma(u(s)) \mathrm{d} W(s) \\
\quad=\int_{0}^{t_{2}}\left(e^{-\left(t_{1}-s\right) A}-e^{-\left(t_{2}-s\right) A}\right) \sigma(u(s)) \mathrm{d} W(s)+\int_{t_{2}}^{t_{1}} e^{-\left(t_{1}-s\right) A} \sigma(u(s)) \mathrm{d} W(s) \\
=\mathrm{III}+\mathrm{I},
\end{aligned}
$$

where III can be estimated first using Burkholder-Davis-Gundy inequality for example:

$$
\left(\mathbb{E}|\mathbb{I}|^{p}\right)^{1 / p} \leq C\left(\int_{0}^{t_{2}}\left(\mathbb{E}\left|\left(e^{-\left(t_{1}-s\right) A}-e^{-\left(t_{2}-s\right) A}\right) \sigma(u(s))\right|_{L_{2}^{0}(H)}^{p}\right)^{2 / p} \mathrm{~d} s\right)^{1 / 2}
$$


and then owing to (3.7), (3.3) and (2.6), we are lead to

$$
\begin{aligned}
\left(\mathbb{E}|\mathbb{I I}|^{p}\right)^{1 / p} \leq & C L_{\sigma}\left(\int_{0}^{t_{2}}\left|\left(e^{-\left(t_{1}-s\right) A}-e^{-\left(t_{2}-s\right) A}\right) A^{\beta}\right|_{L_{2}^{0}}^{2}\left(1+\left(\mathbb{E}|u(s)|^{p}\right)^{2 / p}\right) \mathrm{d} s\right)^{1 / 2} \\
\leq & C L_{\sigma}\left(\int_{0}^{t_{2}}\left(\sum_{i \geq 0} \lambda_{i}^{2 \beta}\left(e^{-\left(t_{1}-s\right) \lambda_{i}}-e^{-\left(t_{2}-s\right) \lambda_{i}}\right)^{2}\right)\left(1+\left(\mathbb{E}|u(s)|^{p}\right)^{2 / p}\right) \mathrm{d} s\right)^{1 / 2} \\
\leq & \frac{C}{2} L_{\sigma}\left(\sum _ { i \geq 0 } \lambda _ { i } ^ { 2 \beta - 1 } \left[2\left(1-e^{-\left(t_{1}-t_{2}\right) \lambda_{i}}\right)-\left(1-e^{-2\left(t_{1}-t_{2}\right) \lambda_{i}}\right)\right.\right. \\
& \left.\left.-\left(e^{-t_{1} \lambda_{i}}-e^{-t_{2} \lambda_{i}}\right)^{2}\right]\left(1+\sup _{t \in[0, T]}\left(\mathbb{E}|u(t)|^{p}\right)^{2 / p}\right)\right)^{1 / 2} \\
\leq & C L_{\sigma}\left(\sum_{i \geq 0} \lambda_{i}^{2 \beta-1}\left(1-e^{-\left(t_{1}-t_{2}\right) \lambda_{i}}\right)\right)^{1 / 2}\left(1+\sup _{t \in[0, T]}\left(\mathbb{E}|u(t)|^{p}\right)^{1 / p}\right) \\
\leq & C^{\prime}\left|t_{1}-t_{2}\right|^{\gamma / 2}\left(1+\sup _{t \in[0, T]}\left(\mathbb{E}|u(t)|^{p}\right)^{1 / p}\right)
\end{aligned}
$$

for some $0<\gamma<1-\alpha-2 \beta$.

We estimate $\mathrm{I}$ in exactly the same way and we get

$$
\left(\mathbb{E}|\mathrm{N}|^{p}\right)^{1 / p} \leq C\left(1+\sup _{t \in[0, T]}\left(\mathbb{E}|u(t)|^{p}\right)^{1 / p}\right)\left|t_{1}-t_{2}\right|^{\gamma / 2}
$$

Eventually, (4.30)-(4.33) yield the result.

Acknowledgements. The author warmly thanks Professor R. Temam for having suggested the study of this problem.

\section{REFERENCES}

[1] A. Bensoussan and R. Temam, Équations stochastiques du type Navier-Stoke. J. Funct. Anal. 13 (1973) 195-222.

[2] J.H. Bramble, A.H. Schatz, V. Thomée and L.B. Wahlbin, Some convergence estimates for semidiscrete Galerkin type approximations for parabolic equations. SIAM J. Numer. Anal. 14 (1977) 218-241.

[3] C. Cardon-Weber, Autour d'équations aux dérivées partielles stochastiques à dérives non-Lipschitziennes. Thèse, Université Paris VI, Paris (2000).

[4] M. Crouzeix and V. Thomée, On the discretization in time of semilinear parabolic equations with nonsmooth initial data. Math. Comput. 49 (1987) 359-377.

[5] G. Da Prato and A. Debussche, Stochastic Cahn-Hilliard equation. Nonlinear Anal., Theory Methods. Appl. 26 (1996) $241-263$.

[6] G. Da Prato, A. Debussche and R. Temam, Stochastic Burgers' equation. Nonlinear Differ. Equ. Appl. 1 (1994) 389-402.

[7] G. Da Prato and D. Gatarek, Stochastic Burgers equation with correlated noise. Stochastics Stochastics Rep. 52 (1995) $29-41$.

[8] G. Da Prato and J. Zabczyk, Stochastic equations in infinite dimensions, in Encyclopedia of Mathematics and its Application. Cambridge University Press, Cambridge (1992).

[9] F. Flandoli and D. Gatarek, Martingale and stationary solutions for stochastic Navier-Stokes equations. Probab. Theory Relat. Fields 102 (1995) 367-391.

[10] I. Gyöngy, Lattice approximations for stochastic quasi-linear parabolic partial differential equations driven by space-time white noise. I. Potential Anal. 9 (1998) 1-25.

[11] I. Gyöngy, Lattice approximations for stochastic quasi-linear parabolic partial differential equations driven by space-time white noise. II. Potential Anal. 11 (1999) 1-37. 
[12] I. Gyöngy and D. Nualart, Implicit scheme for stochastic parabolic partial differential equations driven by space-time white noise. Potential Anal. 7 (1997) 725-757.

[13] I. Gyöngy, Existence and uniqueness results for semilinear stochastic partial differential equations. Stoch. Process Appl. 73 (1998) 271-299.

[14] C. Johnson, S. Larsson, V. Thomée and L.B. Wahlbin, Error estimates for spatially discrete approximations of semilinear parabolic equations with nonsmooth initial data. Math. Comput. 49 (1987) 331-357.

[15] P.E. Kloeden and E. Platten, Numerical solution of stochastic differential equations, in Applications of Mathematics 23, Springer-Verlag, Berlin, Heidelberg, New York (1992).

[16] N. Krylov and B.L. Rozovski, Stochastic Evolution equations. J. Sov. Math. 16 (1981) 1233-1277.

[17] M.-N. Le Roux, Semidiscretization in Time for Parabolic Problems. Math. Comput. 33 (1979) 919-931.

[18] G.N. Milstein, Approximate integration of stochastic differential equations. Theor. Prob. Appl. 19 (1974) 557-562. G.N. Milstein, Weak approximation of solutions of systems of stochastic differential equations. Theor. Prob. Appl. 30 (1985) 750-766.

[19] E. Pardoux, Équations aux dérivées partielles stochastiques non linéaires monotones. Étude de solutions fortes de type Ito. Thèse, Université Paris XI, Orsay (1975).

[20] B.L. Rozozski, Stochastic evolution equations. Linear theory and application to nonlinear filtering. Kluwer, Dordrecht, The Netherlands (1990).

[21] T. Shardlow, Numerical methods for stochastic parabolic PDEs. Numer. Funct. Anal. Optimization 20 (1999) $121-145$.

[22] D. Talay, Efficient numerical schemes for the approximation of expectation of functionals of the solutions of an stochastic differential equation and applications, in Lecture Notes in Control and Information Science 61, Springer, London, (1984) 294-313.

[23] D. Talay, Discrétisation d'une équation différentielle stochastique et calcul approché d'espérance de fonctionnelles de la solution. RAIRO Modél. Math. Anal. Numér. 20 (1986) 141-179.

[24] M. Viot, Solutions faibles aux équations aux dérivées partielles stochastiques non linéaires. Thèse, Université Pierre et Marie Curie, Paris (1976).

[25] J. B. Walsh, An introduction to stochastic partial differential equations, in Lectures Notes in Mathematics 1180 (1986) $265-437$.

To access this journal online:

www.edpsciences.org 